\title{
Improvement of Network Coverage Due to Changes in Array Antennas Parameters
}

\author{
Julian Imami ${ }^{1}$ \\ ${ }^{1}$ Politecnical University of Albania \\ Tirana, Albania \\ julianimami@hotmail.com
}

\begin{abstract}
Telecommunication is one of the most fast changing industries and in the same time with major impact in everyday life of everyone. Due the fact that mobile companies have to compete with each other through offering better services, improvements are always welcomed. The mobile users served by any wireless network need to have at least the required radio coverage to enable the demanded wireless network connectivity while on top the offered network capacity needs to provide the maximum coverage footprint with maximum application throughput. In this paper, we focus on how to optimize a mobile network service performance by fine tuning the antenna azimuth e tilt, such that the geographical coverage area of the antenna can serve more users with better radio conditions. After mentioned changes realized in a specific antenna in the defined region the Key Performance Indicators (KPI) are going to be monitored to identify the improvements made.
\end{abstract}

Keywords: Antennas, Network performance, Azimuth, Tilt.

\section{Introduction}

Telecommunication today is one of the most complexes industries, with the fastest development technology. Technology related to this industry has improved dramatically, impacting in this way all the operators which offer mobile services.

Nowadays even a minor change in one of the many technological aspects can change drastically the service offered. Based on this assumption, this thesis will focus on changes and providing the test results of the modifications for one of the mobile operators which operate in Albania.

Our work that is represented in this paper has come as result of parameter changes of array antennas that are made in the mobile operator taken into account. We know that for a mobile operator antennas are the bases from which different changes can be made. In this paper we have made test related to GSM, UMTS and LTE capabilities.

Changes made consists in azimuth and tilt modifications. These improvements has been made in the third biggest region of Albania in Fier. It has been chosen Fier because based on national statistics this is the third region with the highest density of population in Albania (161 inhabitants for km2) [1].

To verify our method, KPI's such as Access Failure Rate (AFR), Drop Call Rate (DCR), Voice Traffic (Erlang) and Data Traffic $(\mathrm{MB})$ in a live mobile network are measured and compared.

The rest of the paper is organized as follows: In the methodology section are described the changes made in azimuth and tilt. Situation before and after the modifications.

In the network monitoring results section are realized and presented the monitoring of KPIs in the antennas where the changes were realized. The results are presented for network monitoring of situations before and after changes.

In the recommendation part, are suggestions that are made after changes, monitoring of KPIs and analyses of the data collected.

\section{Methodology}

Mobile network operators use radio network planning tools to provide the coverage for the geographical area that a base station (or cells) needs to cover. Turning to the base station antenna, we see that azimuth and tilt are available possible antenna feature to help the mobile network planning needs. The antennas used by the operator are Huawei Agisson antennas ATR451606. They cover 2G, $3 \mathrm{G}, 4 \mathrm{G}$ and $4 \mathrm{G}+$. They are array antennas (pieces of antennas incorporated in the same axe).

The improvements that are made are in two parts: the azimuth in the horizontal part and the tilt in the vertical one. Azimuth refers to the rotation of the whole antenna around a vertical axis. It is the side to side angle. Typically you loosen the main mount bracket 
and swing the whole dish all the way around in a 360 degree circle. [2] Antenna tilt is defined as the angle between the main beam of the antenna and the horizontal plane. [3] The aim of the changes is to have better territory coverage, in the interested area.

After changes in azimuth and tilt we make the same monitoring for every antenna which is in the zone in order to investigate over empty spaces which are without network coverage. We are trying to have a better coverage within the existing antennas, in the case that this improvement is not possible with the existing ones, the only solution is to add a new antenna. When we consider tilt changes, it is important to not increase the part without coverage under the antenna itself.

Changes in azimuth involves horizontal opening of the antenna. For example for 66 degrees main beam for antenna Agisson we set azimuth in 25 degrees. In order that for that direction to have better coverage, it is needed to be 33 degrees in each site of the lobe. After the changes we monitor if there is a good coverage of the entire region. This procedure is made for every single antenna.

The situation before making the changes is represented by an antenna which is placed in a hill with a height of 40 meters. The antenna itself is of 30 meter of heightens. The height of 70 meters is used to cover an inhabited space of approximately $2 \mathrm{~km}$. After improvements through azimuth parameter for the surrounding area, is used tilt modification in order that the coverage is sent as far as possible to cover the region.

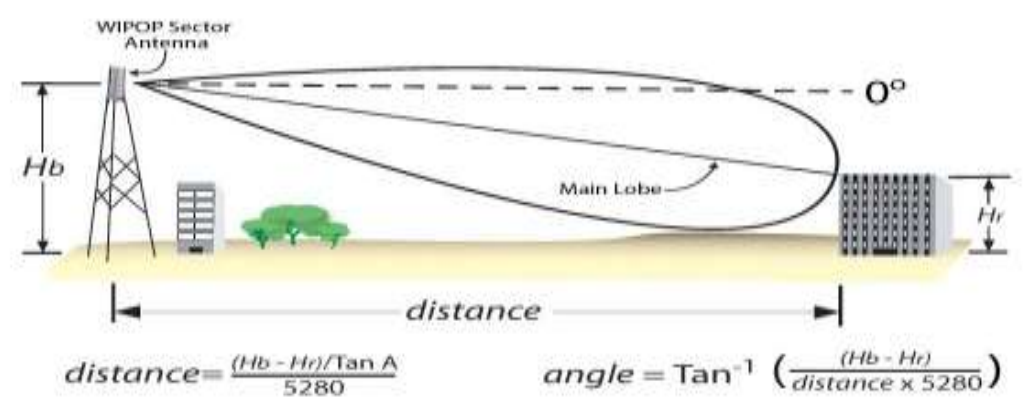

Fig. 1: Antenna Downtilt Example.

Horizon means that the $-3 \mathrm{~dB}$ point on the main lobe shoots off into the horizon and does not touch the earth (assuming flat terrain), Fig.1. The formula for calculating the distance is $((\mathrm{Hb}-\mathrm{Hr}) / \mathrm{Tan} \mathrm{A}) / 5280$ where $\mathrm{A}$ is the angle. The formula for calculating the angle is Tan- $1 *((\mathrm{Hb}-\mathrm{Hr}) /(\mathrm{D} * 5280))$ where $\mathrm{D}$ is the distance.

Tilt calculation is made through a program named Kathrein Scala Division which used formulas as per figure below. The figures 2 and 3 are screens taken from this program which shows our parameters.

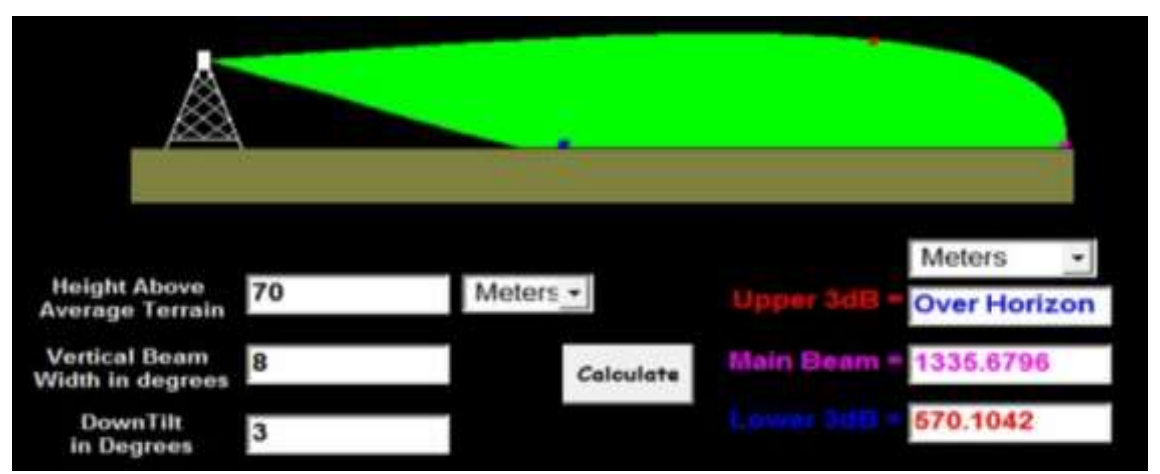

Fig. 2: Information before changes.

As shown in the Fig. 2 we had as parameters: height $70 \mathrm{~m}$, vertical beam approximately 8 and we have set tilt 3 . After the computations we noticed that the upper beam goes toward the horizon, main beam $1.3 \mathrm{~km}$ meanwhile lower $3 \mathrm{~dB}$ approximately 570 meters. Main beam doesn't goes to $2 \mathrm{~km}$ as per our aim. It doesn't cover everything. After the changes made in tilt, we set it to 6 , in order to narrow the space without coverage the situation becomes as per below: 


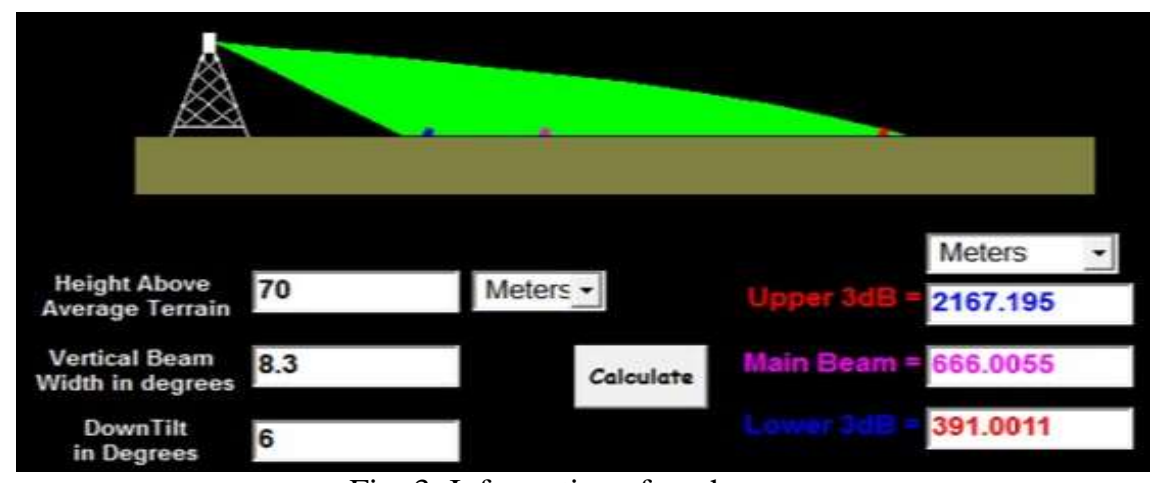

Fig. 3: Information after changes.

In order to have a better narrower antenna network coverage but at the same time to have a better horizontal coverage as well, we change tilt from 3 to 6 . As shown in the Fig. 3 the results are upper $3 \mathrm{~dB}$ goes to approximately $2.1 \mathrm{~km}$, main beam $666 \mathrm{~m}$ and lower $3 \mathrm{~dB}$ to $391 \mathrm{~m}$. This means that we have missing network coverage in the near zone with only $391 \mathrm{~m}$, compared to $570 \mathrm{~m}$ in the first case. Upper is better that our aim is $2.1 \mathrm{~km}$ compared to $2 \mathrm{~km}$ that was our target.

\section{Network monitoring results}

With the given region and the given test appliances, there are made respective monitors and the results are reassumed in the below table. Table 1 shows the results of tests realized before and after changes in azimuth and tilt:

Table 1: KPIs comparison table.

\begin{tabular}{|c|c|c|c|c|c|}
\hline KPI & & \multicolumn{2}{|l|}{ Before } & \multicolumn{2}{|l|}{ After } \\
\hline Strongest Scan $(\mathrm{dBm})[2 \mathrm{G}]$ & Avg. & \multicolumn{2}{|l|}{-53.62} & \multicolumn{2}{|l|}{-53.99} \\
\hline RSCP Scan (dBm) [3G] & Avg. & \multicolumn{2}{|l|}{-75.03} & \multicolumn{2}{|l|}{-72.98} \\
\hline RSRP Scan (dBm) [4G] & Avg. & \multicolumn{2}{|l|}{-93.59} & \multicolumn{2}{|l|}{-94.24} \\
\hline Rx Qual (dedicated) [2G] & Avg. & \multicolumn{2}{|l|}{0.19} & \multicolumn{2}{|l|}{0.2} \\
\hline Ec/Io Scan (dB) [3G] & Avg. & \multicolumn{2}{|l|}{-9.28} & \multicolumn{2}{|l|}{-9.03} \\
\hline RSRQ Scan (dB) [4G] & Avg. & \multicolumn{2}{|l|}{-9.21} & \multicolumn{2}{|l|}{-8.97} \\
\hline Number of Dropped Calls (long call) & Count & \multicolumn{2}{|c|}{0} & \multicolumn{2}{|c|}{0} \\
\hline FTP Throughput DL (Kbps) & Avg. & \multicolumn{2}{|c|}{23466.15} & \multicolumn{2}{|c|}{9467.74} \\
\hline \multirow[t]{2}{*}{ HTTP Download } & $\begin{array}{l}\text { Session } \quad \text { Failure } \\
\text { Ratio }\end{array}$ & \multicolumn{2}{|c|}{0.61} & \multicolumn{2}{|c|}{5.41} \\
\hline & $\begin{array}{l}\text { Mean Throughput } \\
\text { (kbps) }\end{array}$ & \multicolumn{2}{|c|}{12337.76} & \multicolumn{2}{|c|}{6963.32} \\
\hline \multirow[t]{2}{*}{ HTTP Upload } & $\begin{array}{l}\text { Session } \\
\text { Ratio }\end{array}$ & \multicolumn{2}{|l|}{0.07} & \multicolumn{2}{|l|}{0.43} \\
\hline & $\begin{array}{l}\text { Mean Throughput } \\
\text { (kbps) }\end{array}$ & \multicolumn{2}{|c|}{6326.08} & \multicolumn{2}{|c|}{6941.79} \\
\hline DNS Resolution Failure Ratio & Failure & \multicolumn{2}{|l|}{0.09} & \multicolumn{2}{|l|}{0} \\
\hline DNS ResolutionTime (max) & $\mathrm{Sec}$ & \multicolumn{2}{|l|}{0.55} & \multicolumn{2}{|l|}{0.44} \\
\hline \multirow[t]{2}{*}{ Idle Mode (3G Prefered) } & WCDMA & 16117 & $96.08 \%$ & 17705 & $100.00 \%$ \\
\hline & GSM & 657 & $3.92 \%$ & 0 & $0.00 \%$ \\
\hline \multirow[t]{3}{*}{ Idle Mode (4G Prefered) } & LTE & 14101 & $80.30 \%$ & 15989 & $90.24 \%$ \\
\hline & WCDMA & 3311 & $18.86 \%$ & 1729 & $9.76 \%$ \\
\hline & GSM & 148 & $0.84 \%$ & 0 & $0.00 \%$ \\
\hline CSSR (4G prefered) & Successful & 111 & $100.00 \%$ & 162 & $100.00 \%$ \\
\hline
\end{tabular}




\begin{tabular}{|l|l|l|l|l|l|}
\hline & Failed & 0 & $0.00 \%$ & 0 & $0.00 \%$ \\
\hline Handover [2G] & Successful & 126 & $100.00 \%$ & 131 & $100.00 \%$ \\
\cline { 2 - 5 } Inter Frequency Handover [3G] & Failed & 0 & $0.00 \%$ & 0 & $0.00 \%$ \\
& Successful & 3 & $75.00 \%$ & 4 & $100.00 \%$ \\
\cline { 2 - 5 } & Failed & 1 & $25.00 \%$ & 0 & $0.00 \%$ \\
\hline Irat-CS Handover (3G->2G) & Successful & 6 & $100.00 \%$ & 4 & $100.00 \%$ \\
\cline { 2 - 6 } & Failed & 0 & $0.00 \%$ & 0 & $0.00 \%$ \\
\hline Irat-PS Handover (4G->3G) & Successful & 26 & $100.00 \%$ & 7 & $100.00 \%$ \\
\hline & Failed & 0 & $0.00 \%$ & 0 & $0.00 \%$ \\
\hline
\end{tabular}

From the results that are represented in the table we can easily notice that improvements are made in all the cases. In all the monitors the after results are better than in previous situations. For this reason it is recommendable for the company to implement the changes as soon as possible in all the areas that their antennas cover.

\section{Conclusions}

After realizing the monitoring related to the company antennas before and after changes that were made we can conclude by giving the below recommendations:

- In order to improve $3 \mathrm{G}$ coverage in city center, 1 new site is needed.

- FI0003 and FI0007 are partially blocked by new buildings, leading to a limited coverage area, therefore must be relocated.

- To further improvement of $4 \mathrm{G}$ coverage it is needed to implement $4 \mathrm{G}$ in other sites.

In Fig.4 we have a map of Fieri region which is taken from google earth [4]. In this map we have better described where it would be good to implement the changes from above recommendations.

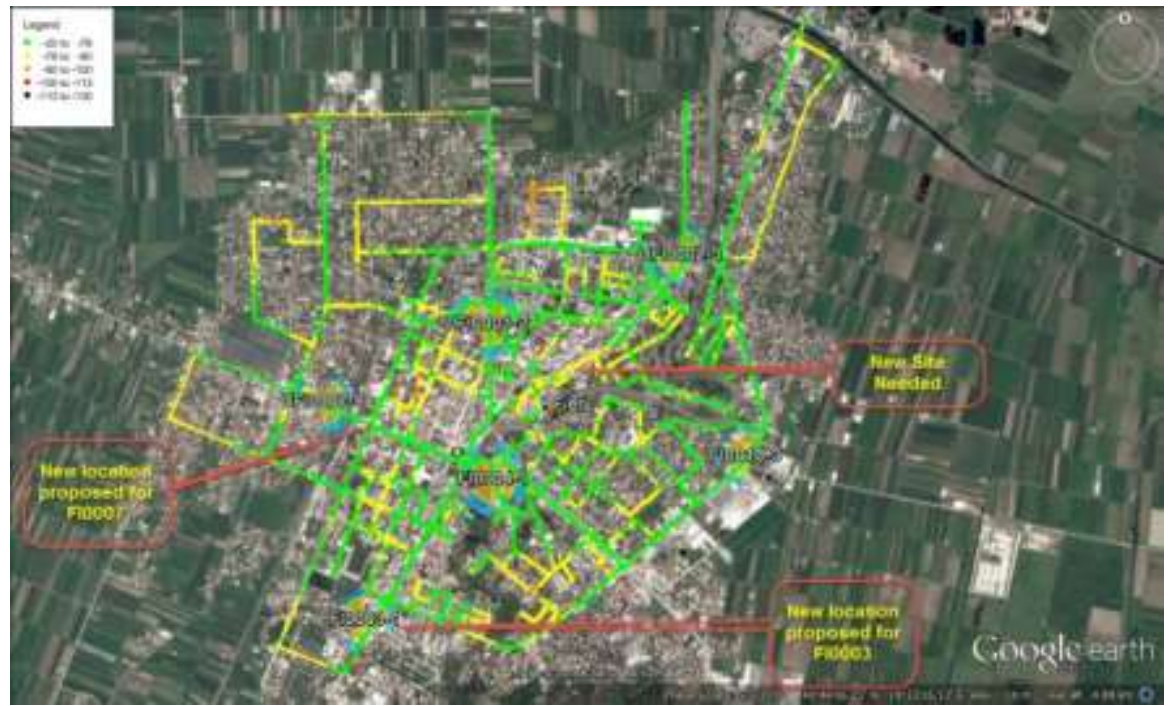

Fig. 4: Fieri region from google earth.

\section{References}

[1] Instituti i statistikave (2017). Vjetari statistikor rajonal, 2017 [Online]. Available: http://www.instat.gov.al/media/3595/vjetaristatistikor-rajonal-shqip-2017-dt13112017.pdf

[2] Statsig (2019, February 12) Explanation of Azimuth and Elevation [Online]. Available: www.satsig.net/azelhelp.htm

[3] Vlad-Ioan Bratu (2012). Self-optimization of Antenna Tilt in Mobile Networks [Online]. Available: https://pdfs.semanticscholar.org/d998/dcd44bffe4d12966e98d9a1c019c29d947d0.pdf

[4] Lars Rasmussen (2005, February 8).Google Maps, Fieri Albania region from google earth [Online]. Available: https://www.google.com/maps 